\title{
Strategies in the evaluation of an on-line computer-assisted unit for intake assessment of mental health patients
}

\author{
DANIEL E. KLINGLER, JAMES H. JOHNSON, and THOMAS A. WILLIAMS \\ Systems and Evaluation Unit, Mental Health and Behavioral Sciences Service \\ Veterans Administration Hospital, Salt Lake City, Utah 84119 \\ and \\ Department of Psychiatry, University of Utah College of Medicine, Salt Lake City, Utah 84112
}

\begin{abstract}
Many applications of on-line computer technology have been blindly accepted as superior to traditional noncomputerized procedures. Strategies utilized in the design of an integrated comparative series of evaluation studies of a prototype computer-assisted psychiatric assessment system are discussed, and preliminary results of several of these evaluation studies are presented.
\end{abstract}

The application of on-line computer systems is a rather recent development in the field of mental health care delivery. The unique capabilities afforded by the application of this technology to intake assessment are only now beginning to be exploited (Johnson, Giannetti, \& Williams, 1976). Questions regarding the advantages of such applications of on-line computer technology remain substantially unanswered. It is our contention that we, as behavioral scientists, must hold ourselves increasingly accountable to the public regarding expenditures of public monies. Thus, we must strive to justify adequately our growing utilization of costly computer systems.

An emerging maxim accompanying the introduction of new methods, technologies, or strategies in health care, research, educational, and other social action programs is that both process and outcome must be measured in order to facilitate the evaluation of alternative programs on the basis of merit. Process studies attempt to insure that a new program has been implemented in accordance with original design specifications upon which funding was provided. Outcome studies attempt to measure the impact of the program upon the target population to which it was addressed (Bernstein \& Freeman, 1975; Caro, 1971; Schaefer, 1973; Suchman, 1967).

Categorization of past evaluative efforts in the field of the behavioral sciences and mental health care delivery is confusing. The range in quality of process and / or outcome measurement procedures is immense

This project has been supported by contracts from the Health Services Research and Development Service, Veterans Administration Central Office, Washington, D.C. Reprint requests should be addressed to Dr. Daniel E. Klingler, Systems and Evaluation Unit, VA Hospital, Salt Lake City, Utah 84113.
(Bernstein \& Freeman, 1975). Garwick (Note 1), presenting what is admittedly only an abbreviated bibliography, lists over 125 program evaluation studies in the field of mental health care delivery. Since the time of his report, many additional mental health care delivery programs and evaluation studies have been funded and implemented. However, the evaluation of applications of computer technology in mental health care delivery is limited mainly to studies of natient management information systems which facilitate record-keeping and operate in a batch processing mode (cf. Kaplan \& Smith, 1974).

Several studies have been reported which have attempted to evaluate computerized assessment systems, but these were not fully automated systems. Weitzel, Morgan, Guyden, and Robinson (1972) compared a structured computerized mental status examination with traditional free-form examinations and found that reports derived from the structured examinations recorded more symptoms and contained fewer omissions. Maultsby and Slack (1971) found that patients enjoyed being interviewed by a computer. Meikle and Gerritse (1970) found that a computerized history resulted in increased numbers of descriptors. Spitzer and Endicott (1969) showed that computer-generated psychiatric diagnosis agreed as well with independently derived clinical diagnosis as did experienced clinicians among themselves. These studies, although important and interesting, do not begin to provide the necessary process and outcome data necessary to justify the application of on-line computer technology to psychiatric intake assessment.

The intent of this paper is to present the rationale for an evaluation strategy, illustrated by preliminary results of several of a series of studies now in progress to evaluate formally the prototype computer-assisted 
on-line psychiatric assessment unit (PAU) which has been in operation for over 2 years at the Veterans Administration Hospital in Salt Lake City.

\section{RATIONALE}

The development of the Utah Project began with a thorough systems analysis of clinical intake decision-making processes. As a result of this analysis, a special unit was formed to perform psychiatric, psychological, social, and physical assessments of all applicants for psychiatric care. All of these assessments were completed with the aid of an on-line computer system. Patient self-report data is collected using on-line keyboard cathode ray tube terminals (CRTs). All clinician and interviewer contacts with patients are structured and computer prompted. The output of this system is a comprehensive patient workup which includes reports of a mental status examination, results of exhaustive diagnostic psychological testing, a social history, a physical examination, and a review of medical systems history. This comprehensive clinical data base is used as intake for decisions concerning patient disposition and treatment. This prototype PAU system has been described in detail elsewhere (Cole, Johnson, \& Williams, 1975; Johnson, Giannetti, \& Williams, 1975; Johnson, \& Williams, 1975; Williams, Johnson, \& Bliss, 1975).

In contrast to the PAU admitting system, most mental health care facilities use an officer-of-the-day, or admitting physician, system for intake triage decision making. Under this traditional scheme, an applicant for care is interviewed briefly by the admitting physician, who usually records an abbreviated history of the present illness, minimal demographic data, the results of a cursory mental status examination, and, if indicated, the results of an abbreviated physical examination. On the basis of this brief intake evaluation, a decision is made to: (1) admit the applicant to an inpatient program for more comprehensive diagnostic evaluation and treatment; (2) refer the applicant to an outpatient program for further evaluation and treatment; (3) prescribe medication; or (4) reject outright the application for care.

The purpose of the present series of studies is to perform a comprehensive comparative evaluation of these two alternative processes for initial patient assessment. In order to compare adequately these processes, it has been necessary to identify and define appropriate operational process and outcome measures which can be expected to be affected by the mode of initial patient evaluation in the mental health care delivery system under study. Answers are sought to the following research questions: (1) Can a psychiatric assessment system which is supported by on-line computer technology provide psychiatric. psychological, social, and physical assessment data at intake which is qualitatively comparable to that provided by an experienced admitting physician and clinical support staff? (2) Can the computer-assisted PAU evaluations be provided at a cost which is competitive with those provided by an admitting physician and support staft? (3) Does completion of a comprehensive intake evaluation at the time of application for care exert a demonstrable impact on the cost and quality of resulting patient care which is comparable or superior to that which follows from the traditional admitting physician intake process?

\section{METHOD}

\section{Experimental Design: Rationale}

In a comprehensive review of outcome studies in mental health care settings, Erickson (1975) found special fault with: (1) the lack of experimental or quasi-experimental design in evaluative studies which often results in serious threats to the internal validity of such studies, and (2) the fact that interpretation of results of evaluation studies of health care delivery is often suspect because but one dependent variable is measured, despite the often-noted lack of correlation among evaluative criteria (May \& Tuma, 1964).

The validity of the results of an evaluation study of a computerized psychiatric assessment unit thus seems to rest upon: (1) the true random assignment of applicants for clinical care to alternative patient assessment processes, and (2) the specification of multiple process and impact measures indicative of the worth of the competing assessment processes.

\section{Experimental Design: Studies in Progrese}

Multiple process variables emerged from the thorough systems analysis requisite for design of the psychiatric assessment unit. Multiple outcome or impact measures have been suggested by Erickson (1975) and Waskow and Parloff (1975). For the Utah Project, variable specification proceeded through the rational approach of eliciting suggestions from senior hospital staff members and cataloging conventionally recognized data collection techniques. Appropriate clinical indicators were then selected, tailored, and quantified by staff members of the project in collaboration with a number of outside consultants from the areas of clinical psychology, psychiatry, and evaluation methodology.

The subjects for the studies now in progress are 195 applicants for admission to the VA Hospital in Salt Lake City. Applicants are assigned on a consecutive basis to one of three experimental groups. Patients assigned to Group 1 receive the entire PAU computer-assisted psychiatric evaluation. Patients assigned to Group 2 are evaluated initially by an admitting physician with over 2 decades of experience in the hospital. He is assisted by any additional personnel he chooses to designate to perform ancillary evaluation functions. Patients assigned to Group 3 are evaluated by both the admitting physician and the PAU system. For Group 3 , the order of evaluation is alternated. Patients whose primary problem relates to substance abuse, including alcoholics, obtunded patients, and those patients who are already receiving treatment at the facility are excluded from the study.

In an attempt to avoid past problems with evaluative research strategies, and to mimic adequately the ideal evaluation paradigm, our process and outcome variable selection scheme resulted in a somewhat novel and complex, but comprehensive, evaluative research plan. The resulting mini experiments are presented in Table 1, categorized by process measurements, impact measurements, and experimental groups utilized. These measures and experiments were selected as representative indicators of the cost efficiency, cost benefit, and quality of the PAU admitting system contrasted with the traditional admitting physician intake process. 
Additional Subjects and Procedures

Because the intent of this paper is to outline a number of alternative strategies in the evaluation of a mental health program, a multifaceted experimental design has been presented. This comprehensive study is now being conducted. In addition, please note that the specific details of subject selection and procedures will be presented together with the preliminary results of the three studies being reported now as illustrations of the overall strategy which is proposed for evaluation of interventionally relevant applications of on-line computer technology in mental health care delivery settings.

\section{PRELIMINARY RESULTS OF ILLUSTRATIVE STUDIES}

Of the mini experiments outlined above, one is complete. In addition, illustrative results of two pilot studies for this project shall be presented. These studies are offered as representative of a wide spectrum of patient assessment process and outcome variables measured or currently under measurement by the staff of the Utah Project.

\section{Utilization of Treatment Staff Time}

This process study is designed to measure the reallocation of staff time and staff resources on an acute treatment ward, which was predicted to result from the implementation of the computerized psychiatric assessment unit. It was hypothesized that the PAU system would significantly reduce the amount of time spent by treatment ward staff in patient assessment functions. By implication, more time could be made available for patient treatment and patient management functions. The activities of

Table 1

Evaluation Studies of the Computerized Psychiatric Assessment Unit (PAU) and the Admitting Physician (MD) Processes

\begin{tabular}{c} 
Study \\
\cline { 2 - 3 } \\
\cline { 3 - 3 }
\end{tabular}

Process Measurement

Assessment Quality

Assessment Format

Assessment Completion Time

Patient Cost

Assessment and Inpatient Care

Treatment Staff Agree-

ment with Disposition

Staff Evaluation and

Treatment Time*

Impact Measurement

Recidivism

Intake Follow-Up Goal

Attainment

Patient-Consumer Satisfaction

Medication Effectiveness

Assessment of Accuracy and

Comprehensiveness of

Clinical Records

$\mathrm{X}$

*This study is longitudinal, covering pre-and postcomputerized PAU implementation.
Table 2

Acute Treatment Ward Work Analysis: Percentage of Time Spent by Ward Personnel on "Intake Workup" Pre- and Post-PAU Installation

\begin{tabular}{lccc} 
& Pre-PAU & Post-PAU & Z \\
\hline $\mathrm{n}$ & 2112 & 3612 & \\
$\begin{array}{l}\text { Percentage Ward Personnel } \\
\text { Time Devoted to "In- } \\
\text { take Workup" }\end{array}$ & 13.5 & 3.6 & $-13.92 *$
\end{tabular}

Note-Work activity units were recorded for each staff member at 15-min intervals over 2-week periods.

${ }^{*} p<.001$

all personnel assigned to the Acute Treatment Ward of the Salt Lake City VA Hospital were subjected to work activity sampling analyses for 2-week periods during May 1974, prior to introduction of the PAU system, and again in July 1975, 9 months following final implementation of all computer-assisted aspects of the PAU system. During the periods of each work-study analysis, all ward personnel recorded their activities on a precoded work log every $15 \mathrm{~min}$. During the initial study, 2,112 work activity entries were recorded; the final study included 3,611 data entries.

Specific work activities were then combined into several general categories such as "intake workup" which included the specific activities "psychiatric history taking and mental status examination," "physical history taking and examination," "psychological testing," "social work history taking," and admission "chart work" by physicians, psychologists, social workers, nurses, nursing assistants, clerks, and trainees of all disciplines. Percentages of time allocated to each general category of activity were then calculated from the data supplied during each of the periods of work-study analysis.

The mean value of percentage time devoted to "intake workup" prior to the implementation of the PAU system was then compared to the mean value of percentage time devoted to this function after the computer-assisted aspects of the PAU system were implemented. Observed differences between mean percentages were tested for significance using a two-tailed $\mathrm{Z}$ test of significance corrected for continuity.

Following the introduction of the PAU system, the amount of time devoted to "intake workup" by the staff of the acute treatment ward decreased from $13.5 \%$ to $3.6 \%$. The differences between "pre-PAU" and "computerized "PAU" were statistically significant $(\mathrm{p}<.001)$, as shown in Table 2.

Quality of Intake Evaluation and Disposition by the PAU vs. the Admitting Physician

The primary hypothesis of this study was that the intake evaluation and disposition performed by the 
Table 3

Percentages of Outpatient Referrals and Agreement by Inpatient Treatment Personnel Regarding "Correctness" of Decision to Admit: PAU System vs. Admitting Physician

\begin{tabular}{|c|c|c|c|}
\hline & PAU & $\begin{array}{l}\text { Admitting } \\
\text { Physician }\end{array}$ & $\mathrm{Z}$ \\
\hline $\mathrm{n}$ & 35 & 27 & \\
\hline Percentage Outpatient Referrals & 40 & 33.3 & n.s. \\
\hline $\begin{array}{l}\text { Percentage Agreement by } \\
\text { Inpatient Staff Regarding } \\
\text { "Correctness" of Decision to Admit }\end{array}$ & 96 & 83 & $-1.32^{*}$ \\
\hline
\end{tabular}

computer-assisted PAU staff would compare favorably with the traditional admitting physician method of intake evaluation and triage decision making.

Sixty-two consecutive applicants for admission to the psychiatric service were divided into two groups. The first group consisted of 35 applicants for treatment; intake evaluation and disposition were completed by PAU staff members. The second group consisted of 27 applicants for treatment; intake evaluation and disposition were completed by a psychiatrist with more than 2 decades of experience at the VA Hospital. The two major reasons for the differing numbers of persons in the two groups were that some patients departed against medical advice in Group 2, and that the availability of an admitting physician was sporadic, determined primarily by interference from competing staff duties. This ex post facto nonexperimental explanation of the group number differences further suggests the superiority of the PAU system.

Comparisons between the two groups were made according to the following measures: (1) the percentage of applicants referred for outpatient treatment, in contrast to the percentage of applicants admitted for inpatient treatment; (2) the agreement between personnel who provided inpatient treatment and the intake evaluator regarding "correctness" of inpatient decisions; and (3) the agreement between the personnel who provided inpatient treatment and the intake evaluator on rated "need for inpatient care."

Analysis of data derived from the 39 patients admitted for inpatient treatment revealed that the group of 21 applicants for care who were evaluated and admitted by the PAU staff did not differ significantly in terms of age or number of previous admissions from the group of 18 applicants for care who were evaluated and admitted by the admitting physician.

From inspection of Table 3, it may be seen that there were no significant differences between the PAU staff and the admitting physician in terms of the percentage of patients referred for outpatient treatment, as opposed to being admitted for inpatient treatment. The PAU staff referred $40 \%$ of the applicants whom they evaluated for outpatient treatment; the admitting physician so referred $33.3 \%$ of the applicanis whom he evaluated. All other patients were admitted for inpatient care.

There was a tendency for the personnel who provided inpatient treatment to agree more often with the inpatient dispositions made by the PAU staff ( $96 \%$ rated as "correct") than with those made by the admitting physician ( $83 \%$ rated as "correct"), but the difference between these percentages failed to reach statistical significance.

Further, from Table 4, it may be seen that inpatient treatment personnel rated "need for in patient care" as slightly greater than did the PAU staff. The mean ratings were 5.0 and 4.8 , respectively, where a rating of " 1 " represents "no need" for inpatient care and a rating of " 7 " indicates "great need" for inpatient care. The difference between these mean ratings was not statistically significant. In contrast. inpatient treatment personnel rated "need for inpatient care" as significantly less than did the admitting physician. The mean ratings were 4.4 and 5.5 , respectively. The difference between these means was statistically significant $(p<.05)$.

\section{Patient-Consumer Satisfaction with the Computerized PAU System}

This pilot study was performed in order to develop measurement techniques for the "patient satisfaction" component of the comprehensive series of evaluation studies. One-hundred and thirty-two consecutive applicants for care were asked to complete consumer satisfaction survey questionnaires comprised of anchored self-report ratings.

The following series of questions was designed to assess the validity of a concern which is prevalent among many providers of health care that patients

Table 4

Rated "Need for lnpatient Care" for Admitted Patients

\begin{tabular}{lcc}
\hline & PAU & Admitting Physician \\
Number of Patients Admitted by Indicated Admissions Process & 21 & 18 \\
Mean of Ratings by Indicated Admissions Process & 4.8 & 5.5 \\
Mean of Ratings by Inpatient Treatment Personnel & 5.0 & 4.4 \\
t Value for Indicated Difference & n.s. & $-1.93^{*}$ \\
\hline
\end{tabular}

Note-A rating of "I" represents "no need" for inpatient care; a rating of " 7 " indicates "great need" for inpatient care.

${ }^{*} p<.05$ 
Table 5

Patient-Consumer Satisfaction With the Computerized PAU Intake System, Expressed as Response Percentages*

Questions
(1) Like Computer Testing
(2) Computer Testing Ton Impersonal
(3) Favor Computerized Intake Testing

$1=$ Hate

$23=$ Indifferent

$7 \quad 5 \quad 219$

$4 \quad 5=$ Liked No Response

$21 \quad 47$

\begin{tabular}{ccc}
\hline Yes & No & No Response \\
\hline 21 & 78 & 1 \\
89 & 11 & 0
\end{tabular}

(4) Computer vs. Interview

Same Worse No Response

(4) Computer vs. Interview

(5) Computer vs. Interview

(6) Like Total Evaluation

\begin{tabular}{|c|c|c|c|c|c|}
\hline & & More Truthful & Same & Less Truthful & No Response \\
\hline & & 46 & 45 & 8 & 0 \\
\hline $1=$ Hate & 2 & $3=$ Indifferent & 4 & $5=$ Liked & No Response \\
\hline 5 & 3 & 29 & 18 & 35 & 11 \\
\hline
\end{tabular}

Note-Row totals differ from $100 \%$ in some cases because of rounding to two digits.

object to what may be viewed as the "dehumanizing" impact of computer testing. The questions addressed included: (1) How do psychiatric patients "like being tested on the computer?" (2) Do they "think computer testing is too impersonal?" (3) Are they "generally in favor of computerized intake testing?" (4) Compared to clinical intake interviews, how well do psychiatric patients like computer testing? (5) Compared to clinical interviews, do psychiatric patients indicate that they are more or less "truthful" in responding to computer testing? (6) How do psychiatric patients like the entire computer-assisted PAU intake evaluation?

The results of this exploratory study are presented in Table 5: (1) Appoximately $68 \%$ of the patients surveyed indicated that they liked being tested on the computer. Approximately $19 \%$ were indifferent to, and approximately $12 \%$ disliked, the procedure. (2) Although approximately $21 \%$ of the patients surveyed indicated that they found computer testing to be "too impersonal," approximately $78 \%$ did not find this to be true. (3) Approximately $89 \%$ of the patients surveyed indicated that they "favored" computerized intake testing. The remaining $11 \%$ did not. (4) Approximately $57 \%$ of the patients indicated that they liked computer testing "better" than clinical intake interviews. Approximately $24 \%$ of the patients preferred the clinical interviews, while $17 \%$ indicated no preference. (5) Compared to clinical intake interviews, approximately $46 \%$ of the patients indicated that they tended to be more "truthful" when responding to the computerized self-report testing. Approximately $45 \%$ indicated that they tended to be as "truthful" in responding to clinical interview or computer testing. The remaining $8 \%$ indicated that they tended to be less "truthful" when responding to computer testing. (6) Only 118 patients (approximately $89 \%$ of the total sample) responded to the question regarding how well they liked the "total [computer-assisted PAU intake] evaluation." Approximately $53 \%$ of the entire sample of 132 patients indicated that they liked their evaluation. Approximately $29 \%$ indicated that they were indifferent to it, while $8 \%$ disliked the evaluation process.

Although none of these results, taken singly, represents overwhelming support for the computerized PAU intake assessment process, the results appear to indicate that most patients do react quite favorably to the computerized procedures.

\section{CONCLUSIONS}

From these studies, it would appear that implementation of the computerized on-line psychiatric assessment system now in operation at the VA Hospital in Salt Lake City has reduced and redistributed patterns of work loads among intake and treatment personnel. These preliminary results also indicate that treatment dispositions rendered by the PAL compare favorably with those rendered by an experienced admitting physician. Further. patients seem quite favorably disposed toward computerassisted on-line psychiatric assessment as an alternative to clinical interviews. However, the preliminary results of these studies are not offered as a detinitive evaluation of the prototype, computerassisted psychiatric intake assessment process. Rather, they are presented as what we feel to be necessary facets of a comprehensive evaluation paradigm adequate to identify properly the process and outcome strengths and weaknesses of computerized vs. noncomputerized systems for intake assessments of applicants for psychiatric care.

Clear deficiencies have existed in past evaluation studies of applications of computer technology to 
clinical assessment processes. As the initial step in any proper evaluative effort, we suggest a systems analytic dissection of the specific process which has been computerized. Based upon this analysis, a rigorous experimental design should be developed. Appropriate measures of process and outcome should then be identified and developed in order to assess both the cost efficiency and the cost benefit of the new application of computer technology.

It is our contention that the benefits of this systematic and comprehensive analytic and design specification approach to evaluation should be generalizable to all applications of on-line computer technology in the field of mental health care delivery.

\section{REFERENCE NOTES}

1. Garwick, G. A working bibliography of program evaluation: Report of the Program Evaluation Project. Minneapolis, Minnesota, October 1971.

\section{REFERENCES}

Bernstein, I. L., \& Freeman, H. E. Academic and entrepreneurial research. The consequences of diversity in federal evaluation studies. New York: Russel Sage Foundation, 1975.

CARo, F. G. (Ed.) Readings in evaluation research. New York: Russel Sage Foundation, 1971.

Cole, E. B., Johnson, J. H., \& Williams, T. A. Design considerations for an on-line computer system for automated psychiatric assessment. Behavior Research Methods \& Instrumentation, 1975, 7, 195-198.

ERICKSON, R. C. Outcome studies in mental hospitals: A review. Psychological Bulletin, 1975, 82, 519-540.

Johnson, J. H., Giannetti, R. A., \& Williams, T. A. Realtime psychological assessment and evaluation of psychiatric patients. Behavior Research Methods \& Instrumentation, $1975,7,199-200$.
Johnson, J. H., Giannettr, R. A., \& Williams, T. A. Computers in mental health care delivery: A review of the evolution toward interventionally relevant on-line processing. Behavior Research Methods \& Instrumentation, 1976. 8. 83-91.

Johnson, J. H., \& Williams, T. A. The use of on-line computer technology in a mental health admitting system. American Psychologist, 1975, 30, 388-390.

Kaplan, J., \& Smith, W. An evaluation program for a regional mental health center. In Crawford, J. L.. Morgan, D. W.. \& Gianturco, D. T.. (Eds.), Progress in mental health information systems: Computer applications. Cambridge, Massachusetts: Ballinger Publishing Company, 1974.

Maultsby, M. C., \& Slack. W. V. A computer-based psychiatry history system. Archives of General Psychiatry. $1971,25,570-572$.

May, P. R. A., \& Tuma, A. H. Choice of criteria for the assessment of treatment outcome. Journal of Psychiatric Research. 1964, 2, 199-209.

MeIKLE, S., \& GerRitse, R. A comparison of psychiatric symptom frequency under narrative and checklist conditions. American Journal of Psychiatry, 1970, 127, 155-158.

SCHAEFER, M. Evaluation/decision-making in health planning and administration. Chapel Hill. North Carolina. University of North Carolina. Report prepared for the Health Services and Mental Health Administration, 1973. (NTIS No. PB-234 841)

Spitzer, R. L., \& Endicott, J. DIAGNO II: Further developments in a computer program for psychiatric diagnosis. American Journal of Psychiatry, 1969 (Supp.), 125, 12-21.

Suchman, E. A. Evaluative research. Principles and practice in public service and social action programs. New York: Russel Sage Foundation. 1967.

Waskow, I. E., \& Parloff, M. B., (Ed.). Psychotherapy Change Measures. Rockville, Maryland: National Institute of Mental Health, 1975. [DHEW Publication No. (ADM) 74-120]

Weitzel, W. D., Morgan, D. W., Guyden, T. E.. \& Robinson, J. A. Towards a more efficient mental status examination. Archives of General Psychiatry, 1972, 28, 215-218.

Williams, T. A., Johnson, J. H., \& Bliss, E. L. A computer-assisted psychiatric assessment unit. American Journal of Psychiatry, 1975, 132, 1074-1076. 